\title{
Permanence et évolution dans la Revue d'économie Industrielle : trente ans de publications
}

Richard Arena et Cindy Navarro

\section{(2) OpenEdition \\ 1 Journals}

Édition électronique

URL : https://journals.openedition.org/rei/4172

DOI : $10.4000 /$ rei.4172

ISSN : 1773-0198

Éditeur

De Boeck Supérieur

\section{Édition imprimée}

Date de publication : 15 juin 2010

Pagination : 381-401

ISSN : 0154-3229

\section{Référence électronique}

Richard Arena et Cindy Navarro, «Permanence et évolution dans la Revue d'économie Industrielle: trente ans de publications », Revue d'économie industrielle [En ligne], 129-130 | 1er et 2e trimestres 2010, document 17, mis en ligne le 15 juin 2012, consulté le 02 juin 2022. URL : http://

journals.openedition.org/rei/4172 ; DOI : https://doi.org/10.4000/rei.4172 


\section{PERMANENCE ET ÉVOLUTION DANS LA REVUE D'ÉCONOMIE INDUSTRIELLE: TRENTE ANS DE PUBLICATIONS

Mots-clés : Économie industrielle, histoire des revues, découpage productif, approches théoriques, domaines de recherche

Key words : Industrial Organization, History of Journals, Industrial Sections, Theoretical Approaches, Research Areas

$\mathrm{L}$

'objet de cette contribution est d'utiliser le support des contributions parues dans la Revue d'Économie Industrielle pendant trente années (1977-2007) pour offrir au lecteur un panorama de l'évolution en France de l'économie industrielle comme discipline durant la période considérée.

Deux thèmes majeurs sont ici privilégiés : la différenciation des principaux domaines de recherche qui composent l'économie industrielle et la pluralité des approches théoriques qui ont été mobilisées par les auteurs des travaux recensés. Bien évidemment, dans les deux cas, le biais retenu est celui du regard de la Revue sur la discipline dont elle se réclame. Ainsi, nous ne prétendons évidemment pas proposer un bilan qui se voudrait neutre. La Revue ne peut être considérée a priori comme un baromètre qui mesurerait fidèlement les changements caractéristiques de l'économie industrielle dans les trente ans considérés.

(*) Les auteurs souhaitent remercier les participants au colloque organisé à Nice-Sophia Antipolis à l'occasion des trente ans de la Revue d'Économie Industrielle pour leurs critiques, remarques et suggestions; les insuffisances de cette contribution sont cependant de leur seule responsabilité. 
Elle est d'abord une revue francophone et occupe donc un espace international différent de celui des publications de langue anglaise. Sa création accompagna cependant la naissance même et le développement de l'économie industrielle moderne en France. On peut en effet dater des années 1970 cette émergence marquée aussi à la même époque par la constitution de l'ADEFI (Association pour le Développement des Études sur la Firme et sur l'Industrie) et du premier GRECO (aujourd'hui on parle de GDR...) d'économie industrielle par le CNRS. En ce sens la Revue s'inscrit sans aucun doute dans l'histoire de la discipline en France.

La Revue n'est pas non plus un lieu dans lequel toutes les approches représentatives de l'économie industrielle en France sont présentes à égalité. Elle a d'abord été associée - à tort ou à raison - à ce que certains appelaient il y a deux ou trois décennies l' «École française d'économie industrielle». Elle est depuis de plus en plus devenue un carrefour - et, nous l'espérons un lieu de dialogue pluraliste - des approches théoriques dont les représentations et les explications des phénomènes industriels entrent parfois, elles aussi, en concurrence au plan intellectuel et scientifique. Mais elle ne prétend pas non plus que ce carrefour ou ce lieu soit toujours neutre ou équitable.

Enfin, la réalité industrielle a connu ces dernières années de profonds changements, liés à l'émergence de l'économie numérique, à bien d'autres innovations technologiques radicales mais aussi à la globalisation économique. Le domaine et les frontières de l'économie industrielle ont ainsi fortement évolué et la Revue, malgré ses efforts constants d'actualisation, n'a certainement pas pu tenir compte entièrement de cette évolution.

La présente contribution ne peut donc offrir qu'un regard - parmi d'autres sur les changements qui ont marqué la discipline dans les trente dernières années. Nous pensons cependant que ce regard reflète ceux d'un grand nombre de chercheurs et de groupes de chercheurs en France et ailleurs et qu'à ce titre déjà, il mérite d'être caractérisé.

\section{I. - LES DOMAINES DE RECHERCHE EN ÉCONOMIE INDUSTRIELLE ET LA REI (1977-2007)}

Le choix des différents domaines de recherche retenus au sein de la discipline a résulté d'un compromis entre plusieurs exigences. Il fallait d'abord que ces domaines couvrent l'ensemble du champ de l'économie industrielle dans le contexte des économies développées de la période considérée. Ainsi, un domaine comme celui de la planification de l'industrie n'a pas été retenu en tant que tel. Il fallait ensuite qu'ils ne soient pas étrangers à la Revue. Ainsi, un domaine comme celui de la petite entreprise ou celui de l'entreprise artisanale n'a pas été distingué en raison de sa très faible représentativité dans l'ensemble des articles de la Revue. Il a été inclus dans le domaine « Économie de la firme et de l'organisation de l'entreprise », alors même que dans une revue italienne par 
exemple, il serait impensable de ne pas distinguer le thème spécifique de la petite entreprise en raison des spécificités structurelles du système productif italien. Il fallait enfin que le nombre des domaines soit assez grand afin d'obtenir une diversification significative de leurs contenus respectifs.

Ces contraintes ont permis de faire apparaître douze domaines particuliers: " Méthodes et théorie de l'économie industrielle », "Systèmes productifs, branches d'activité et relations inter-entreprises », " Marchés, concurrence et politiques anti-trust », "Économie de la firme et de l'organisation des entreprises », « Économie industrielle régionale et spatiale », " Économie industrielle et réseaux », «Économie de la connaissance », « Économie de la technologie », «Économie industrielle internationale », et enfin « Autres domaines de l'économie industrielle ».

Observons donc comment l'importance relative de ces domaines a évolué entre 1997 et 2007. Pour cela, nous avons construit des graphiques qui montrent quel pourcentage occupe chaque année chacun de ces domaines dans l'ensemble des articles publiés.

\section{Méthodes et théorie de l'économie industrielle}

Cette rubrique fait l'objet du graphique 1

FIGURE 1 : Méthodes et théorie de l'économie industrielle

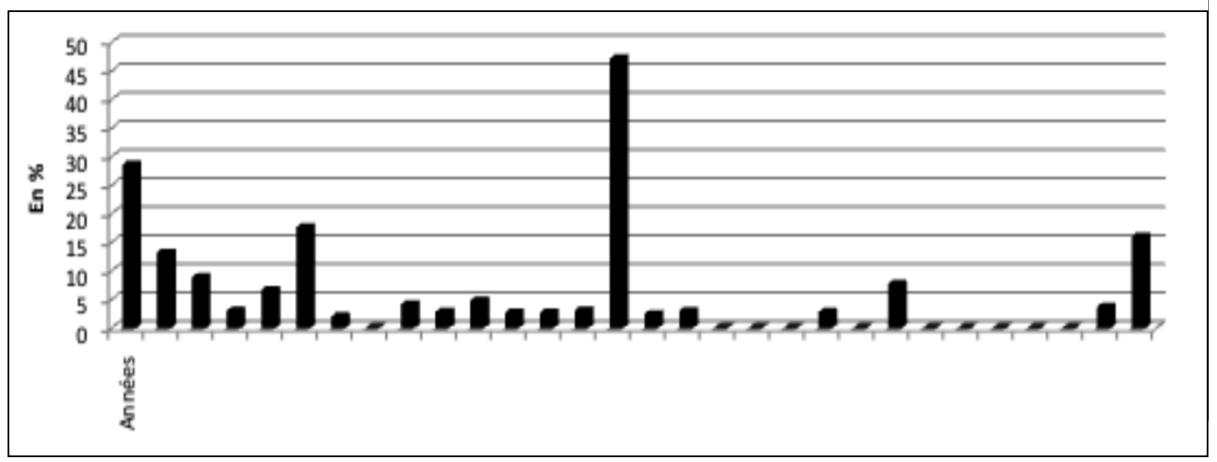

Celui-ci met en évidence un déclin lent mais continu des préoccupations méthodologiques et théoriques dans le domaine des publications de la Revue. On peut ainsi observer pendant les cinq premières années d'existence de la Revue une sorte de période de découverte de la discipline, alors nouvelle pour la France. Durant cette période initiale, les auteurs tentent d'explorer les concepts de base, les principales approches existantes mais aussi les limites de la discipline (notamment Morvan 1977, Rainelli 1977, Hénin 1978, Morvan et Marchesnay 1979, Encaoua et Héon 1979, Dupuy et Marchesnay 1981, Marchesnay 1984). Après ce processus de reconnaissance du champ et des méthodes, l'intérêt pour les préoccupations théoriques et méthodologiques se réduit ou plutôt se normalise. 


\section{FIGURE 2 : Systèmes productifs, branches d'activité,}

relations inter-entreprises

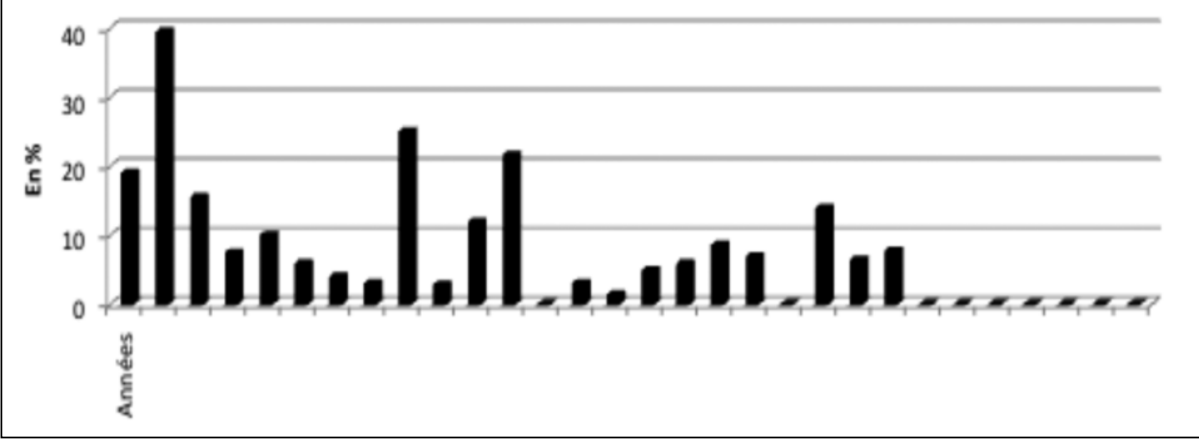

Cette réduction est lente et elle traduit les préoccupations naturellement empiriques de l'économie industrielle. L'objet de cette dernière consiste davantage en une explication des phénomènes observés dans la réalité historique qu'en la construction détaillée d'un programme de recherches théorique dont les étapes abstraites seraient la première préoccupation. Par ailleurs, on assiste probablement à partir de la fin des années 1980 à la mise en place des principaux courants qui forment aujourd'hui encore la « boîte à outils de la discipline » (par exemple, néo-institutionnalisme, évolutionnisme ou recours privilégié à la théorie des jeux), et qui tantôt s'opposent et tantôt se recoupent.

\section{Systèmes productifs, branches d'activité et relations inter-firmes}

Comme le montre le graphique 2 , jusqu'à la fin des années 1980 , ce domaine joue un rôle fondamental dans les préoccupations des articles publiés par la Revue.

C'est alors la période où un point de vue « mésoanalytique » ou parfois même « structuraliste » l'emporte sur un point de vue plus « comportementaliste » ou au moins « microéconomique » dans la discipline. Il ne s'agit pas alors principalement de privilégier l'étude de la rationalité et des stratégies des firmes mais plutôt de partir des "systèmes productifs » nationaux et/ou régionaux vus comme des ensembles cohérents de relations interindustrielles ou inter-entrepreneuriales. Le concept de « découpage productif » (incluant les branches d'activité, les « sections », les secteurs, les filières ou parfois des regroupements ad hoc) fait alors l'objet de fortes préoccupations car il apparaît comme la clé et le fondement des explications du mode de fonctionnement du système productif ( $\mathrm{cf}$ par exemple Rainelli 1977, numéro sur la statistique industrielle 1978, Humbert 1978, Gillard 1978, Toledano 1978, Parent 1979, Rainelli 1979, Stoffaes 1980, Humbert 1980, numéro spécial sur les politiques industrielles 1983, numéro spécial sur les restructurations industrielles 1984, Reynaud- Cressent 1985). 
Marchés, concurrence et politiques anti-trust

\section{FIGURE 3 : Marchés, concurence et politiques antitrust}

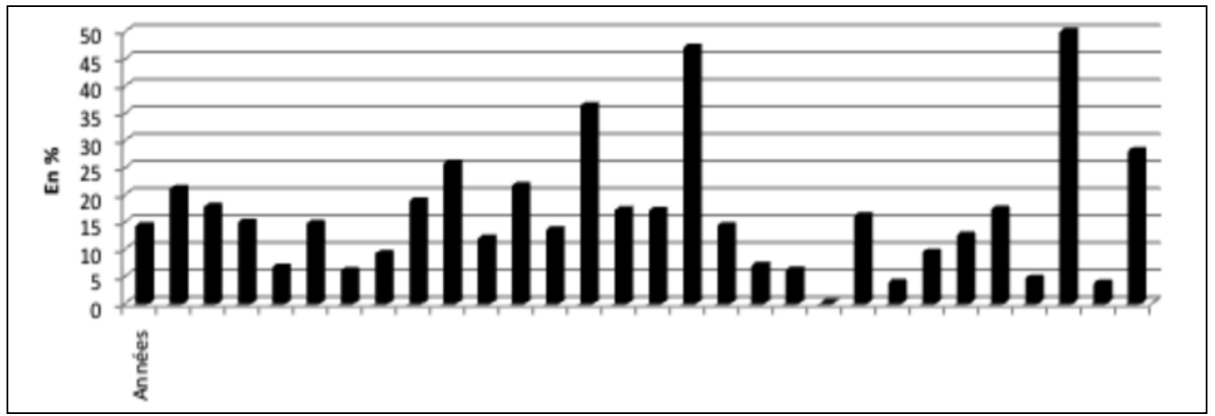

Ce dernier traduit la permanence de sa présence mais aussi de sa place substantielle dans l'ensemble des contributions à la Revue. Cette constatation est loin d'être surprenante. Le thème évoqué constitue le « cœur » traditionnel de l'économie industrielle. Pour certains économistes, on pourrait même écrire qu'il s'identifie à cette discipline. C'est pourquoi, malgré quelques fluctuations en termes de pourcentage, l'ensemble « Marchés, concurrence et politique anti-trust » ne perd jamais longtemps son poids tout à fait essentiel.

\section{Économie de la firme et de l'organisation des entreprises}

Cette thématique qui fait l'objet du graphique 4 est elle aussi permanente et c'est tout naturel.

Toutefois, on observe que jusqu'à la fin des années 1980, son importance relative est assez faible. Cette observation est sans doute à mettre en relation avec le poids corrélatif occupé par le domaine « Systèmes productifs, branches d'activité et relations inter-firmes » pendant la même période. On a là une confirmation de la prédominance des approches dites « structurales » sur les approches « comportementales » dans cette partie de l'histoire de l'économie industrielle

\section{FIGURE 4 : Économie de la firme et de l'organisation de l'entreprise}

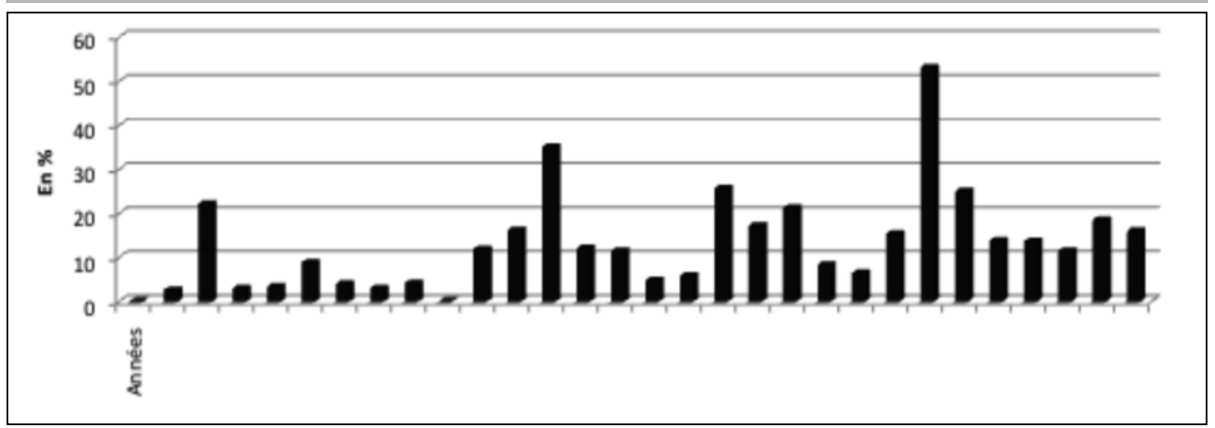


en France. Le niveau de la firme était jugé comme peu pertinent quand il s'agissait de comprendre les problèmes fondamentaux de la discipline.

En revanche, après 1987-1988, la situation se modifie profondément. Le deuxième grand ouvrage de Williamson, The Institutions of capitalism (1) et les deux livres de Aoki, The cooperative game theory of the firm et The economic analysis of the Japanese firm paraissent en 1984 et 1985. La Revue publie d'ailleurs en 1988 une recension écrite par Éric Brousseau intitulée « De nouvelles approches de l'entreprise » (Brousseau, 1988) dans laquelle ces trois ouvrages sont considérés, ainsi que des contributions de la décennie précédente, en particulier de Kenneth Arrow et Herbert Simon. Cette année-là marque un tournant puisqu'après 1988, la place des contributions à l'économie de la firme et de l'organisation de l'entreprise s'avère beaucoup plus substantielle et demeurera désormais à un très haut niveau: le thème de l'économie de l'entreprise reste permanent mais devient une préoccupation centrale de la Revue.

\section{Économie industrielle régionale et spatiale}

Comme le montre le graphique 5 ci-après, les dimensions spatiale et régionale de l'économie industrielle mobilisent de façon permanente l'attention des contributeurs de la Revue.

FIGURE 5 : Économie industrielle régionale et spatiale

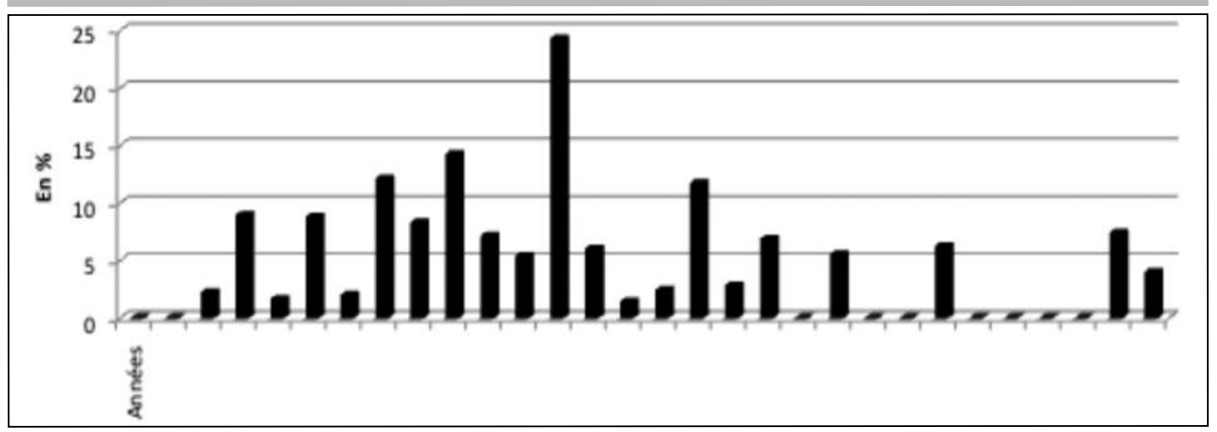

Toutefois, la montée en puissance du domaine est très progressive et connaît une poussée en 1989 pour ensuite s'inverser. Après cette date, pendant un certain nombre d'années, le domaine disparaît entièrement, comme à la fin des années 1990 ou entre 2001 et 2005. En outre, lorsqu'il est présent, le domaine perd l'importance qu'il a connue dans les années 1980. Deux facteurs peuvent expliquer ces constatations. Le premier est l'intérêt que suscite chez les économistes appliqués les effets des lois Defferre de 1982 sur la Décentralisation pendant l'ensemble des années 1980; il était donc naturel que l'étude des dimensions spatiales de l'économie industrielle retienne davantage l'attention.

(1) Un compte rendu critique du premier ouvrage de Williamson - Markets and Hierarchies - avait été écrit pour la Revue en 1979 par Robert Reix (Reix, 1979) 
Le deuxième facteur est lié à l'émergence de la Nouvelle Économie Géographique au milieu des années 1990 et au fait qu'elle a très fortement contribué à renouveler mais aussi à spécialiser l'économie spatiale au sein du champ de l'analyse économique; l'économie industrielle «spatiale»a alors vu corrélativement décroître son importance relative.

\section{Économie industrielle et réseaux}

Le thème de l'économie industrielle des réseaux est entièrement inexistant dans les années 1970 et 1980, comme le montre le graphique 6.

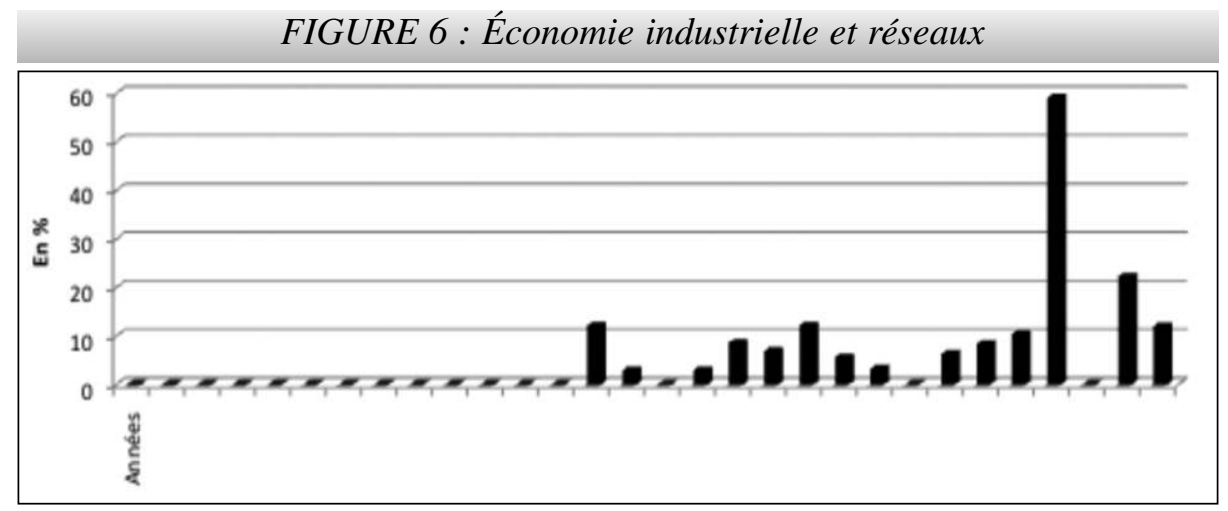

Ce n'est qu'à partir du tout début de 1990 que les préoccupations relatives aux réseaux s'affirment, que ce soit dans le domaine des grands réseaux industriels (type électricité), des communautés d'entreprises, des réseaux sociaux, des réseaux numériques ou encore très simplement de la théorie des réseaux. Cette tendance ne se traduit pas par l'apparition d'un domaine prédominant mais une certaine permanence apparaît qui est loin d'être négligeable.

\section{Économie de la connaissance}

On trouve dans le graphique 7 une situation assez comparable à celle de l'économie des réseaux.

\section{FIGURE 7 : Économie de la connaissance}

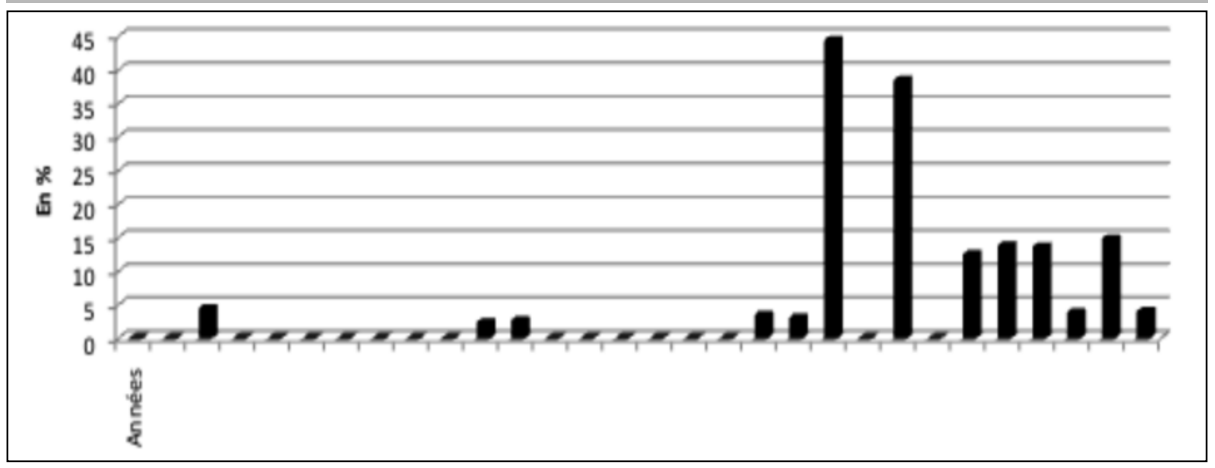




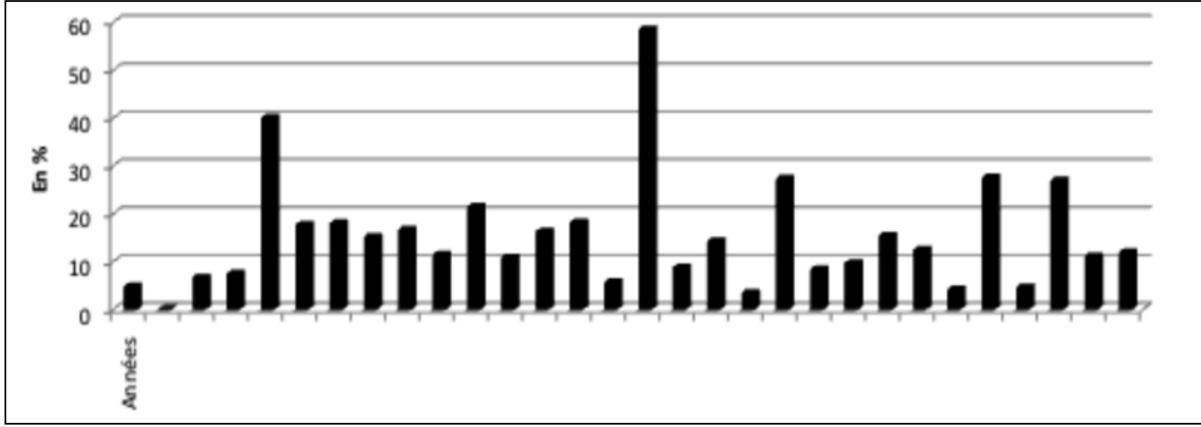

En effet, les contributions relatives à l'économie de la connaissance sont pratiquement inexistantes avant 1995-1996, alors qu'elles prennent une grande place à compter de 1997 et pendant toute la dernière décennie. Ces observations sont le résultat de plusieurs facteurs. Le premier est évidemment lié à l'impact des technologies de l'information et de la communication sur les modes d'échange, de production et de distribution qui exerce ses effets les plus sensibles à partir du début des années 1990. Le deuxième est l'impact de la Déclaration de Lisbonne de mars 2000 dans les débats publics. Le troisième est l'émergence de l'économie de la connaissance entendue non comme un objet de recherche mais comme une démarche particulière d'appréhension des phénomènes dits industriels.

\section{Économie de la technologie}

Le graphique 8 ne requiert pas beaucoup de commentaires.

Il confirme en effet que le changement technologique a toujours constitué une préoccupation permanente du programme de recherches de l'économie industrielle, même si sa signification diffère selon la période considérée.

\section{Économie industrielle internationale}

Le graphique 9 (page suivante) montre lui aussi une certaine permanence des préoccupations des dimensions internationales de l'économie industrielle.

Cette permanence ne doit pas cependant occulter l'existence de deux périodes distinctes. Jusqu'à 1987 , le domaine est très présent et substantiel. Après 1987, la présence s'estompe un peu jusqu'à faire apparaître des années « creuses » sans aucune contribution à la Revue, notamment depuis 2003. La raison de ces évolutions ne saurait tenir à l'inactualité de l'économie industrielle internationale mais à un phénomène analogue à ce qui avait été observé à propos de l'économie régionale et spatiale. La thématique considérée tend à susciter de moins en moins d'articles dans la Revue. L'explication tient là aussi 


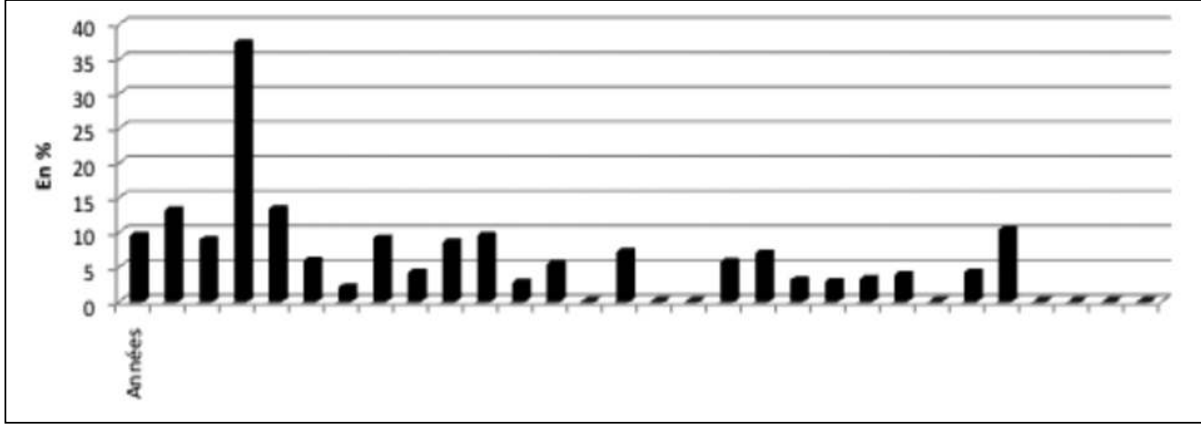

à une spécialisation croissante de l'économie internationale, processus qui conduit les auteurs à orienter davantage leurs publications vers des revues spécialisées en économie internationale.

\section{Économie industrielle et environnement}

Le graphique 10 se suffit à lui-même.

Le thème de l'environnement est très sous-estimé par la Revue. Seuls, des numéros spéciaux et/ou thématiques ont été consacrés à ce domaine.

\section{Politiques économiques}

Comme le montre le graphique 11 (page suivante), le thème des politiques économiques, qu'elles soient industrielles ou macroéconomiques, est loin d'être négligeable.

Il est assez dépendant de l'actualité. Ainsi, les années 1980 constituent une période qui a attiré les contributeurs dans le domaine particulier de la politique industrielle. Le contexte s'y prêtait: la politique industrielle avait alors été remise à l'ordre du jour, notamment en relation avec l'idée de reconstruction

\section{FIGURE 10 : Environnement}

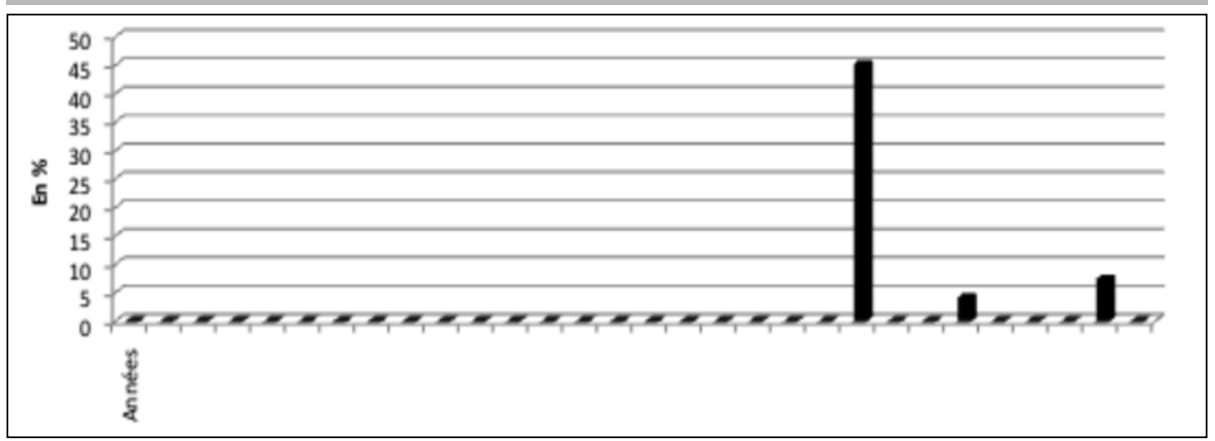




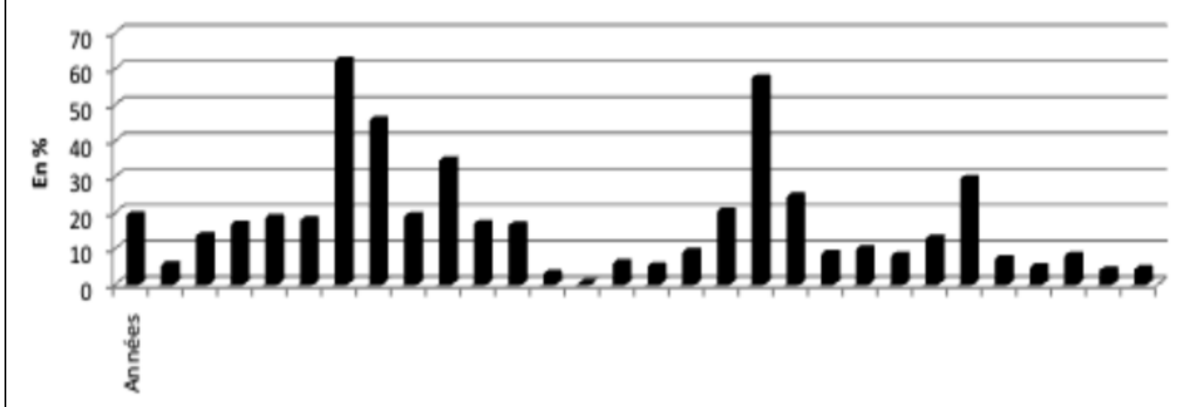

de certaines des filières technologiques du pays. Depuis la fin de ces années, les préoccupations de politique économique existent toujours mais elles deviennent plus spécifiques. L'intérêt pour la politique de la concurrence, par exemple, s'accroît et le poids relatif du thème fluctue et diminue à la fois.

\section{II. - LES PRINCIPALES APPROCHES EN ÉCONOMIE INDUSTRIELLE ET LA REI (1977-2007)}

Nous considérerons dans un deuxième temps la diversité des approches telle qu'elle apparaît dans les grandes périodes de la vie de la Revue depuis sa création. Cette diversité reflète à la fois les caractéristiques idiosyncrasiques du contexte français mais aussi les tendances principales qui se sont manifestées au plan international.

La première période d'existence de la Revue (la fin des années 1970 et le premier tiers des années 1980): les préoccupations programmatiques et statistiques

Les préoccupations programmatiques qui caractérisent cette période d'existence de la Revue sont exprimées clairement dès son premier numéro, en particulier dans les articles de Morvan (Morvan, 1977) et de Rainelli (Rainelli, 1977), puis dans une note très significative de Marchesnay et Morvan (1978). On y trouve notamment une réflexion sur la démarche méso-analytique et sur la « dimension intermédiaire » que cette dernière semble nécessairement impliquer.

Cette démarche est présentée comme une solution alternative à la dichotomie micro/macro et aux limites qu'elle rencontre en matière d'agrégation et d'incompatibilité logique entre les deux niveaux. Son objectif « est de posséder une méthode capable d'expliquer le fonctionnement du système productif et de répondre à des questions du genre: quel type de concurrence régule les rapports entre les unités ? Pourquoi les entreprises se développent-elles dans telle ou telle direction? Qu'est ce qui explique le choix de la diversification? de l'intégra- 
tion? » (Marchesnay et Morvan, $1978: 100$ ). Trois « propositions » (ibid.) résument cette démarche. La première ne consiste pas à bâtir un artefact statistique moyen de façon arbitraire mais à trouver un schéma explicatif qui autorise l'interaction des niveaux micro et macro-économiques. La deuxième consiste à introduire une « logique de l'intention » qui permettrait d'expliquer comment des firmes actives sont susceptibles de modifier un environnement global contraignant mais suffisamment flexible pour leur permettre de mettre en ouvre leurs stratégies propres. La troisième enfin vise à abandonner les démarches fondées sur l'équilibre et l'optimisation pour leur préférer celles qui autorisent l'adaptation et l'évolution continues (à nouveau, Marchesnay et Morvan, ibid.).

Ces «propositions » ont parfois fourni les fondements de ce que l'on a pu appeler l' « École française d'Économie Industrielle ( Palloix et Rizopoulos, 1977 : 9). Se fondant sur la démarche méso-analytique que l'on vient d'évoquer, cette École, très présente, voire prédominante dans la première période d'existence de la Revue a en fait utilisé la distinction conceptuelle entre structures, comportements et performances, afin de lui conférer une signification très différente de celle des défenseurs, le plus souvent anglo-saxons, de ce paradigme. C'est d'ailleurs ce que Morvan note dans l'article programmatique paru dans le premier numéro de la Revue et déjà évoqué ici: " $\mathrm{La}$ séquence structures $=>$ stratégies $=>$ performances n'attire pas $(. .$.$) à ce point$ les analystes français, beaucoup plus soucieux de partir quelque peu à la découverte et se refusant à adopter une méthode réductrice qui rejetterait de l'observation les faits qui n'entreraient pas dans les schémas théoriques usuels » (Morvan, 1977 : 15). On retrouve ici le projet fondamentalement inductif de l'École française que Morvan exprimait à sa façon en opposant « le modèle abstrait de concurrence » à une représentation plus réaliste ou plus ancrée dans l'observation, la « concurrence contemporaine », version française de la notion de « concurrence praticable » introduite par J.-M. Clark (Morvan, 1976, chapitre II).

Cette préférence pour l'approche inductive explique les préoccupations statistiques très présentes dans les publications de la première période d'existence de la Revue. Un bon exemple que l'on peut citer à l'appui de cette remarque est le numéro spécial consacré à la statistique industrielle dans la Revue et paru en 1978. Il faut lui ajouter la même année divers articles consacrés aux indicateurs et aux outils de mesure notamment en matière de relations entre efficacité et rentabilité (numéro spécial consacré à ces relations, 1978). Plus généralement, ces préoccupations traduisent ce qui pouvait alors être caractérisé comme une certaine capillarité entre les chercheurs de l'INSEE et les économistes industriels français.

D'un côté, les économistes universitaires qui publiaient dans la Revue souhaitaient mieux appréhender - y compris sur le plan de la mesure empirique - ce « niveau intermédiaire » que nous avons déjà évoqué. Ils s'intéressaient donc énormément aux représentations du système productif et de ses découpages ainsi que de l'interdépendance qui les liait. À titre d'exemple, le lecteur pourra se référer à l'échange entre Gillard et Rainelli relatif à l'appréhension et à la mesure des découpages productifs (Rainelli 1977, Gillard 1978, Rainelli 1979). 
D'un autre côté, les économistes de l'INSEE tentaient alors de comprendre la signification de la fin des « Trente Glorieuses », les chocs pétroliers des années 1970 et ce qu'ils caractérisaient alors comme la « rupture de 1974 ». Plus précisément, ils avaient publié en 1975 la « fresque historique du système productif » (Division « Étude des entreprises », 1975, Guibert B. et alii, 1975) et introduit en 1981 la notion de « crise du système productif » (Dollé, 1979; Varii autores, 1980; INSEE, 1981 et Dutailly, 1981). Or, ils pensaient alors pouvoir compléter leurs recherches en s'appuyant sur ceux des économistes universitaires pour essayer d'expliquer cette crise car ils avaient à faire face à des « difficultés » de compréhension « redoutables quand il [s'agissait] d'analyser la période récente » (Dutailly, 1981).

Tout naturellement, les préoccupations des économistes industriels et des économistes de l'INSEE ont donc convergé, au moins partiellement, et c'est ce que traduisent bien certains des numéros de la période considérée.

La deuxième période d'existence de la Revue (la fin des années 1980): la politique et la dynamique industrielles

Le projet de l'École française va peu à peu s'affaiblir dans les années 1980. Plusieurs indices l'indiquent.

Tout d'abord, dans un article présenté à un colloque dès 1981 et publié en 1983, Marchesnay posait déjà la question de l'état du projet mésoanalytique (Marchesnay, 1983). Sa réponse était prudente mais elle exprimait des inquiétudes d'abord liées « aux pesanteurs théoriques, aux modes de pensée acquis » (Marchesnay, 1983 :17) qui avaient tendance à enfermer les représentations des économistes au sein des catégories traditionnelles de la micro et de la macroéconomie. D'autres « pesanteurs » étaient évoquées par l'auteur, en commençant par celles qui sont liées aux « organismes officiels de collecte des données »: «il n'existe toujours pas de statistiques des groupes, de nomenclature de filières ». Il était enfin fait référence à celles qui « viennent enfin du pouvoir politique, qui élabore une politique économique largement fondée sur une certaine idée du système productif » (Marchesnay, 1983 : 18).

Le contenu de la première édition du Traité d'Économie Industrielle (Arena et alii, 1988) qui date de 1988 traduit bien - lui aussi - l'effritement progressif du projet d'école française. Ce traité inclut certes des contributions d'auteurs censés appartenir à cette école (notamment J. De Bandt, M. Marchesnay ou Y. Morvan) mais dans la préface de 1988, les responsables de l'ouvrage (L. Benzoni, J. De Bandt, P.M. Romani et R. Arena) notent que les " démarches retenues dans ce traité se caractérisent par leur pluralité et leur variété » (Arena et alii, 1988 : IV). Les influences de l'approche évolutionniste et de la démarche néo-institutionnaliste alors assez récentes se font davantage sentir que celle d'une possible «École française », à travers des auteurs tels que Foray, Gaffard, Garrouste, Guilhon, Le Bas, Quelin, Queré ou Zuscovitch, en particulier dans le chapitre 7 consacré au thème "Systèmes productifs et technologie ». La préface de la deuxième édition est, quant à elle, clairement pessimiste concernant l'avenir du projet d'une École française: «Le projet - 
parfois formulé - de bâtir une économie industrielle "à la française" semble aujourd'hui de plus en plus problématique bien que certains continuent à défendre un tel programme de recherches » (Arena et alii, 1991 : III).

Enfin, dans un texte de 1999 intitulé « Un changement dans l'orientation de la Revue d'Économie Industrielle », Arena constate l'échec du projet d'École française, même si les questions posées par ce projet restent pertinentes et ne sont toujours pas résolues: «Il faut bien convenir que l'EFEI [École Française d'Économie Industrielle - RA et $\mathrm{CN}$ ] a échoué dans sa tentative d'inscrire les stratégies des firmes au sein de découpages productifs non marchands. Elle a certes décrit, caractérisé chacun de ces niveaux ou découpages, tant au plan analytique que sur le terrain empirique (...). La description ne saurait suffire. L'EFEI n'a pas fourni d'explication ou d'analyse générale de la manière dont la coordination s'effectue au sein de découpages différents du marché. La question demeure cependant posée et garde sa signification: comment les firmes peuvent-elles inscrire leurs stratégies au sein de découpages auxquels elles appartiennent? Par exemple, la notion de stratégie de filière a-t-elle un sens? »(Arena, 1999 : 19).

Cet affaiblissement du projet de l'EFEI se reflète tout naturellement dans le contenu de la Revue, comme l'a montré notre première partie: c'est là la principale raison qui explique le recul accéléré du domaine "Systèmes productifs, branches d'activité et relations inter-firmes » après le milieu des années 1980 dans l'ensemble des publications de la Revue.

En liaison avec cet affaiblissement, la deuxième période de l'histoire de la Revue se caractérise par l'accent mis sur les notions de politique et de dynamique industrielles.

Pour ce qui est de la politique industrielle, la première partie de cette contribution a déjà mis en évidence l'accroissement substantiel des contributions intervenu dans les années 1980 en raison de la place nouvelle accordée à l'intervention économique de l'État à cette époque en France. Les thèmes évoqués évoluent cependant avec la conjoncture politique nationale et européenne. C'est ainsi que l'accent mis sur la politique de développement des filières au début des années 1980 se déplace vers la politique de la concurrence ou la politique d'aide aux PME dix ans plus tard (numéros spéciaux sur la politique de la concurrence, 1993 et 1995 ou sur les PME/PMI, 1994): l'Union européenne se préoccupe davantage de réguler la concurrence que de faire jouer à l'État un rôle directeur dans le cadre des restructurations de l'industrie nationale.

Pour ce qui est de la dynamique industrielle, il est frappant de constater que, pendant la période 1989-1992, quatre contributions sont toutes consacrées à l'élaboration de ce programme de recherches possible (De Bandt, 1989; Gaffard, 1990; Arena, 1990; Carlsson, 1992). À chaque fois, l'objectif est de construire une théorie dans laquelle la référence à l'équilibre cesse d'être centrale, laisse la place au concept d'évolution et permet de mieux comprendre les changements qui caractérisent les firmes et les technologies. La conception de J. De Bandt est la plus proche de celle du projet méso-analytique de l'École française, tandis que celle de J.-L. Gaffard est certainement celle qui en est le plus éloignée; elle admet en effet la nécessité de construire une micro- aussi bien qu'une macro- 
économie de l'innovation mais n'opte pas pour l'élaboration d'un niveau « intermédiaire ». L'origine du poids de ces préoccupations de dynamique industrielle au sein de la Revue se trouve certainement dans les recherches de Bo Carlsson (Carlsson, 1987 et 1989) qui faisaient alors l'objet d'une très grande attention parmi les contributeurs et les lecteurs. Au-delà de ces quatre articles, la Revue publie dans la même période un numéro spécial sur l'investissement (numéro sur l'investissement, 1987) et un numéro spécial sur l'organisation et la dynamique industrielle (numéro correspondant, 1990), qui confirment l'importance du domaine de la dynamique industrielle dans la période.

La troisième période d'existence de la Revue (le tournant des années 1990): l'émergence de nouveaux courants théoriques et de nouvelles questions empiriques

Les années 1990 constituent un vrai tournant pour la Revue.

En premier lieu, l'émergence de nouveaux courants la conduit en effet à s'éloigner de plus en plus de sa matrice initiale constituée par la mésoanalyse, puis par ce que J. De Bandt appelait de manière très significative l' « approche mésoéconomique de la dynamique industrielle », pour accueillir à sa manière trois nouveaux courants théoriques essentiels pour le développement de l'économie industrielle.

Le premier est celui de la théorie des jeux, d'abord signalé par un article de Gisèle Umbhauer (Umbhauer, 1988) qui caractérise en 1988 de manière synthétique les contributions de Jacquemin (1985), Ponssard(1988), Schmalensee (1988), Tirole (1988) et du GREMAQ (1988). La Revue publie au départ relativement peu d'articles utilisant la théorie des jeux mais comme le montre le graphique suivant, à partir de 1988, le nombre d'articles publié indiqué en ordonnée fait apparaître une tendance lente mais régulière à la hausse, même si ce dernier reste globalement faible sur l'ensemble de la période.

On notera en particulier que l'année 2007 ne doit pas être considérée comme le signe d'une brutale accélération de la tendance. En fait, les années qui suivent révèlent à nouveau un niveau de bas étiage. Simplement, en 2007, la

Évolution de la théorie des jeux dans la Revue d'Économie Industrille (1977-2007)

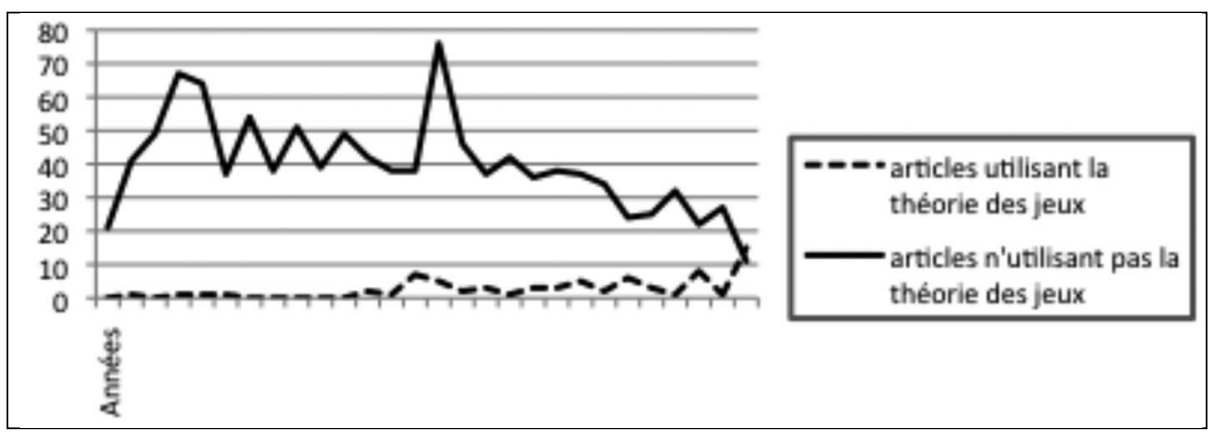


publication d'un numéro spécial consacré aux problèmes d'interaction informationnelle dans la théorie des jeux fausse un peu les résultats.

Le deuxième courant qu'il convient de mentionner est celui de l'évolutionnisme. Vers la fin des années 1980, plusieurs articles paraissent, dont l'inspiration est clairement évolutionniste comme par exemple les textes de Foray (1989), Gaffard (1989), Le Bas (1990), Foray (1990). La tendance s'accélère à partir de 1992 avec la parution d'un numéro spécial consacré à la diversité technologique en Europe (numéro spécial « Diversité technologique et cohérence en Europe », 1992 incluant l'ensemble des fondateurs du courant évolutionniste en matière de changement technologique (notamment Bellandi, Cohendet, Dosi, Foray, Llerena, Lundvall ou Metcalfe), puis ne cessera de se renforcer jusque - à titre d'exemple - l'interview de R. Nelson (Krafft et Maupertuis, 1996). Dès 1990 J. De Bandt avait d'ailleurs considéré l'évolutionnisme comme l'un des courants de recherche compatible avec la dynamique industrielle (De Bandt, 1990).

Un troisième courant est celui du néo-institutionnalime. Nous avons déjà noté dans la première partie de ce travail comment la recension de Brousseau de 1988 avait en quelque sorte inauguré la prise en compte par la Revue de ce courant. Comme on l'a noté, le nombre des articles de facture néo-institutionnaliste ne cessera par la suite de se renforcer. La participation de Coase à l'École Méditerranéenne d'Économie Industrielle alors associée à la Revue (septembre 1991) ainsi que l'obtention du prix Nobel par cet auteur (octobre 1991) vont constituer une nouvelle occasion (De Bandt, Romani, Torre, Ravix, 1991) d'évoquer ce courant autour de ce prix et de la publication en français d'un de ses textes (Coase, 1991). On signalera aussi à titre d'exemple l'article de Foss de 1994 (Foss, 1994) qui propose de construire un «pont» théorique entre les perspectives évolutionnistes et néo-institutionnalistes. L'importance de ce courant dans la Revue sera enfin matérialisée par un très beau numéro double consacré en 2000 à l'Économie des Contrats, introduit par Brousseau et Glachant et incluant deux articles de Coase et Williamson (numéro spécial «Économie des contrats: bilan et perspectives, $2000 »)$.

En second lieu, notre troisième période s'est caractérisée par l'émergence de nouveaux thèmes empiriques qui ont considérablement modifié la perception que l'on pouvait se faire de l'économie industrielle jusque-là. Pour le dire autrement, de nouveaux secteurs d'activité ont vu le jour au sein de nos économies qui annonçaient une transformation profonde de la manière dont la production et la distribution des biens et des services se déroulent. Nous nous référerons ici à trois exemples significatifs.

Le premier est celui des prémisses de ce qui va devenir l'économie fondée sur la connaissance. Ainsi, en 1987, la Revue joue à plein son rôle de veille en publiant un numéro spécial consacré aux «nouvelles industries de l'information et de la communication », présenté par Humbert et introduit par Alain Madelin, alors ministre de l'Industrie, dans lequel on voit a posteriori se constituer ce que sera l'économie numérique du futur (numéro spécial « Les nouvelles industries de l'information et de la communication », 1987). En 
1997, elle publiera dans la même veine un autre numéro spécial consacré à l' ' économie industrielle de la science » (numéro spécial «L'économie industrielle de la science », 1997), préfacé par Callon et Foray (Callon et Foray, 1997) avec une interview d'Hubert Curien, ancien ministre de la Recherche par J. De Bandt (De Bandt, 1997): dans cet ouvrage, les problèmes du savoir comme bien public, des droits de propriété intellectuelle et de brevets sont largement abordés et préfigurent eux aussi les questions que se posent aujourd'hui les analystes de l'économie numérique.

Le deuxième exemple auquel nous nous référerons est l'accent mis sur les activités de l'immatériel qui annoncent elles aussi le développement futur de l'économie numérique contemporaine. On peut citer ici à titre d'exemple le numéro spécial de 1988 sur les services à l'industrie et la chronique de 1996 de J. De Bandt consacrée aux services informationnels qui soulignent l'importance cruciale de la tertiarisation dans l'évolution industrielle mais surtout le « poids de la composante informationnelle » (De Bandt, 1996 : 79) au sein de cette tendance de longue période. À beaucoup d'égards, ce deuxième exemple rejoint le premier car, comme lui, il préfigure l'émergence des économies fondées sur la connaissance.

Le dernier exemple que nous considérerons est celui des contraintes environnementales de la dynamique industrielle. C'est là un sujet fort important qui a donné lieu lui aussi à un numéro spécial, introduit par Mondello (numéro spécial correspondant, 1998). On peut cependant regretter que - comme cela a été déjà indiqué dans la première partie de cette contribution - ce thème pourtant novateur n'ait pas beaucoup suscité d'échos ultérieurs.

\section{La période d'existence la plus récente de la Revue (les années 2000): économie de la connaissance et interactions sociales}

Il est évidemment beaucoup plus difficile de parler d'une période qui est très récente et pour laquelle nous manquons en partie de recul pour proposer une interprétation. Cependant, deux tendances semblent incontestables.

D'un côté, la période est clairement marquée par le débat autour de la notion d' " économie fondée sur la connaissance (knowledge-based economy) ", comme l'illustre la section consacrée à ce thème dans la première partie de ce travail. En effet, l'émergence des nouvelles technologies de l'information et de la communication n'a pas seulement été à l'origine d'importants changements technologiques dans les firmes. Elle a bouleversé les modes d'information, de communication et d'organisation au sein des firmes et sur les marchés. Ces bouleversements ont eu des effets considérables sur les recherches menées au sein de l'économie industrielle appliquée mais ils ont aussi conduit à des modifications et de nouvelles avancées de la théorie de l'économie industrielle. Parmi ces dernières, il convient en particulier de souligner le fait que la notion de connaissance diffère de celle d'information et rend ainsi possible le transfert d'éléments de cognition par le biais d'interactions sociales plus larges et plus riches que de simples interactions stratégiques. Il est donc naturel que, parmi les avancées matérialisées dans la Revue, on trouve un certain nombre 
de travaux portant sur la prise en compte de formes d'interaction sociale dans l'échange autres que celles que proposent les théories de l'équilibre partiel ou général ou encore la théorie des jeux classique avec connaissance commune.

Au titre de l'économie de la connaissance, on ne sera donc pas étonné de voir mentionner ici le numéro spécial de 2001 intitulé « What policies in support for RD? » (numéro spécial, 2001), le numéro spécial de 2002 portant sur « les droits de propriété intellectuelle » introduit par Coriat (numéro spécial, 2002), le numéro spécial intitulé « Connaissance et économie industrielle » introduit par Kogut et Dibiaggio (numéro spécial, 2005) et surtout le remarquable numéro spécial de 1999 ( EÉconomie de la connaissance ») introduit par Dibiaggio (numéro spécial, 1999). Ces numéros évoquent bien sûr les réalités empiriques qui caractérisent les économies fondées sur la connaissance (les deux premiers mentionnés notamment) mais ils discutent aussi (en particulier, le dernier) des évolutions théoriques qui s'avèrent nécessaires pour mieux comprendre ces réalités. L'économie de la connaissance occupe donc une place centrale dans la Revue pendant les années 2000, comme l'attestent aussi les numéros ordinaires publiés dans la période.

Corrélativement, au titre des formes d'interaction sociale, il convient de mentionner trois numéros spéciaux importants. Le premier date de 2003. Il concerne la "morphogenèse des réseaux » (numéro spécial correspondant, 2003) et analyse comment l'économie des interactions peut faire apparaître des formes de réseaux différents selon que l'on a affaire à un « cadre global et statique », " global et dynamique », « local et statique » ou « local et dynamique » (Cohendet, Kirman et Zimmermann, 2003). On peut aussi rapprocher de ce numéro un article de 2001 de Deroïan et Zimmermann (2001) qui concernait aussi la formation des réseaux. Le deuxième numéro concerne la « dynamique des marchés » (numéro spécial correspondant, 2004). Introduit par Arena, Longhi et Psillaki, il étudie comment les arrangements institutionnels et réglementaires, les dispositifs informationnels, les formes d'interaction sociale et les modes de formation et de variation des prix qui permettent de réguler les marchés conduisent à des fonctionnements différents qu'il convient de spécifier et d'étudier, établissant ainsi la nécessité de remplacer l'idée d'un marché universel par celles de la diversité et de l'analyse typologique des marchés. Enfin, le dernier numéro que nous évoquerons concerne le thème « Processus de contagion et interactions stratégiques » (numéro spécial correspondant, 2006). Introduit par Solal et Tallon, il consiste à faire porter l'attention sur les formes d'interaction sociale qui correspondent à des « contagions », entendues soit comme des contagions entre croyances, soit comme des contagions entre actions et qui relèvent de modes d'émergence épistémiques ou évolutionnaires (Solal et Tallon, 2006 : 8). Comme on peut le noter, les numéros évoqués analysent une question que les économistes industriels ont trop souvent sous-estimée et qui s'impose nécessairement dans une économie fondée sur la connaissance: comment penser la coordination d'agents ou de firmes dans une économie où les interactions ne se réduisent pas à un mécanisme d'enchères centralisé mais impliquent le plus souvent des transferts de connaissance explicite ou tacite entre acteurs? 
$\mathrm{Si}$, dans la période la plus récente, les thèmes que nous venons de recenser s'avèrent dominants, comme le montrent les graphiques de la partie 1 consacrés à l'économie de la connaissance et à l'économie des réseaux, ils ne résument cependant pas toute la production existante. Les autres courants évoqués dans la partie précédente continuent à susciter l'intérêt des auteurs de la Revue et d'autres courants sont également présents comme par exemple des formes d'institutionnalisme différentes de celle de type williamsonien (on pense notamment au numéro spécial "Organisations et institutions », 2001). Cette remarque permet de comprendre qu'il devient aujourd'hui de plus en plus difficile d'assimiler la Revue à un courant théorique particulier comme on aurait pu être tenté de le faire au moment de sa création. Elle est donc devenue un carrefour de débats plutôt que le lieu de mise en œuvre d'un programme de recherche particulier.

\section{III. - REMARQUES CONCLUSIVES}

Cette contribution à l'histoire de la Revue d'Économie Industrielle ne prétend pas à l'objectivité. L'un de ses deux co-auteurs a en effet lui-même été un acteur dans cette histoire et il ne peut donc avoir fait entièrement abstraction de cette expérience. Nous pensons toutefois que deux conclusions peuvent être tirées de notre analyse, sans risque majeur d'être contredits.

En premier lieu, la Revue a fortement évolué depuis sa création. Au départ, son projet se confondait à la fois avec celui de l'économie industrielle ellemême au moins dans le cadre français et partiellement avec celui d'une école de pensée en construction. Avec le passage du temps, cette assimilation entre les trois projets est devenue de moins en moins crédible. D'une part, le champ de l'économie industrielle s'est progressivement élargi et complexifié et le projet théorique initial s'est avéré de moins en moins apte à rendre compte des réalités nouvelles dont l'émergence a accompagné ce processus. D'autre part, la Revue s'est de moins en moins identifiée à un seul projet. Comme on l'a conclu plus haut, elle n'est plus - à supposer qu'elle l'ait jamais été - la revue d'un programme de recherche spécifique. Elle est devenue de plus en plus un carrefour d'accueil d'approches diverses. Cette situation n'est pas seulement le résultat d'une adaptation. Il correspond aussi à un souhait qui avait été exprimé par l'un des co-auteurs de ce travail il y a environ dix ans dans un article de la Revue intitulé «Un changement dans l'orientation de la Revue d'Économie Industrielle » (Arena, 1999); la Revue, disait le texte, « doit continuer à accompagner, voire même à précéder les évolutions de la discipline et c'est pourquoi son comité de rédaction a décidé à la fois d'élargir son objet de recherche à toutes les activités liées à la production des firmes et d'accueillir plus particulièrement les nouvelles approches qui ont vu le jour ces dernières années ». (Arena, 1999 : 8-10). C'est ce que la Revue a essayé de faire sans doute insuffisamment depuis 1999.

En second lieu, le pluralisme de la Revue nous semble conforme à ce qu'est l'économie industrielle aujourd'hui, c'est-à-dire, un champ où aucune théorie ne peut aujourd'hui prétendre dominer et expliquer l'ensemble des problèmes 
qui se posent à la discipline. L'impression qui prévaut est même inverse; elle est celle d'un domaine balkanisé où chaque courant semble se spécialiser autour d'un ensemble spécifique de questions. Pour le dire autrement, dans le domaine de l'économie industrielle, nous sommes bien loin d'une "synthèse » et il faut pour le moment nous contenter d'explications partielles, parfois difficiles à intégrer.

\section{BIBLIOGRAPHIE}

ARENA R. (1990), « La dynamique industrielle: tradition et renouveau », Revue d'Économie Industrielle, vol. 53, troisième trimestre.

ARENA R. (1999), «Un changement dans l'orientation de la Revue d'Économie Industrielle », Revue d'Économie Industrielle, vol. 87, $\mathrm{n}^{\circ} 1$.

ARENA R., BENZONI L., de BANDT J., ROMANI P.M. (sous la responsabilité de) (1988), Traité d'Économie Industrielle, Economica, Paris; deuxième édition, 1991.

CALLON M. et FORAY D. (199), «Introduction: nouvelle économie de la science ou socioéconomie de la recherche scientifique », Revue d'Économie Industrielle, vol. 79, premier trimestre.

CARLSSON B. (1987), "Reflections on industrial dynamics: the challenges ahead ", International Journal of Industrial Organization, 5(2).

CARLSSON B. (sous la responsabilité de) (1989), Industrial dynamics: technological, organizational and structural changes in industries and firms, Kluwer Academic Publishers, Boston.

CARLSSON B. (1992), «Industrial dynamics: a framework for analysis of industrial transformation », Revue d'Économie Industrielle, vol. 61, troisième trimestre.

COASE R. (1991), «L'organisation industrielle: un programme de recherches », Revue d'Économie Industrielle, vol. $58, \mathrm{n}^{\circ} 1$, quatrième trimestre.

COHENDET P., ZIMMERMANN J.-B., KIRMAN A. (2003), «Émergence, formation et dynamique des réseaux: modèles de la morphogenèse », Revue d'Économie Industrielle, vol. 103 , deuxième et troisième trimestres.

De BANDT J. (1977), « Les structures industrielles dans le cadre de la division internationale du travail », Revue d'Économie Industrielle, vol. 2, quatrième trimestre.

De BANDT (1996), «Industrie: la dynamique industrielle par les services informationnels », Revue d'Économie Industrielle, vol. 49, troisième trimestre.

De BANDT (1997), «Interview d'Hubert Curien, ex-ministre de la Recherche », Revue d'Économie Industrielle, vol. 79, premier trimestre.

De BANDT J., ROMANI P.M., TORRE A., RAVIX J.-L. (1991), « Ronald H. Coase, prix Nobel 1991 de sciences économiques, ou de l'autre côté du "tableau noir"”, Revue d'Économie Industrielle, vol. $58, \mathrm{n}^{\circ} 1$, quatrième trimestre.

DEROÏAN F. et ZIMMERMANN J.-B. (2001), «Cumul d'influence et réseaux sociaux: une application aux processus de diffusion des innovations », Revue d'Économie Industrielle, vol. 96, premier trimestre.

Division « Étude des entreprises » (1975), « Fresque historique du système productif », collection de l'INSEE, série $\mathrm{E}, \mathrm{n}^{\circ} 27$.

DOLLÉ M. (1979), «L'impact de la crise sur les branches industrielles », Économie et Statistiques, $\mathrm{n}^{\circ} 108$, février.

DUPUY Y. et MARCHESNAY M. (1982), «Les dimensions de l'économie industrielle », Revue d'Économie Industrielle, vol. 21, troisième trimestre.

DUTAILLY J.-C., (1981) : « La crise du système productif », Économie et statistiques, vol. 138

ENCAOUA D. et HÉON M., (1979) : " Groupes de société et structure industrielle », Revue d'Économie Industrielle, vol. 9, troisième trimestre.

FORAY D., (1989) : «Les modèles de compétition technologique: une revue de littérature », Revue d'Économie Industrielle, vol. 48, deuxième trimestre. 
FORAY D., (1990) : «Recherche et technologie militaires: la remise en cause d'un modèle », Revue d'Économie Industrielle, vol. 21, troisième trimestre.

FOSS N., 1994 : "Why transaction costs economics need evolutionary economics », Revue d'Économie Industrielle, vol. 68, deuxième trimestre.

GAFFARD J.-L., (1989) : " Marchés et organisation dans les stratégies technologiques des firmes industrielles », Revue d'Économie Industrielle, vol. 48, deuxième trimestre.

GAFFARD J.-L., (1990) : «Introduction » du numéro spécial « Organisation et dynamique industrielle », Revue d'Économie Industrielle, vol. 51, premier trimestre.

GILLARD L., (1978) : « Nouvelles réflexions sur les découpages industriels », Revue d'Économie Industrielle, vol. 6, quatrième trimestre.

GREMAQ Antoine Augustin, (1988) : Dynamique, information incomplète, stratégies industrielles, Economica, Paris.

GUIBERT B. et alii, (1975) : « La mutation industrielle de la France », collection de l'INSEE, série $\mathrm{E}, \mathrm{n}^{\circ}$ 31-32.

HÉNIN P. Y., (1978) : « De l'efficacité à la rentabilité; jalons pour un programme de travail », Revue d'Économie Industrielle, vol. 5, troisième trimestre.

HUMBERT M., (1978) : «Concurrence et hiérarchie inter-industrielle », Revue d'Économie Industrielle, vol. 5, troisième trimestre.

HUMBERT M., (1980) : «Évolution récente des théories de la division internationale du travail », Revue d'Économie Industrielle, vol. 14, quatrième trimestre.

INSEE, (1981) : La Crise du système productif, INSEE, Paris, décembre.

JACQUEMIN A., (1985) : Sélection et pouvoir dans la nouvelle économie industrielle, Economica, Paris et Cabay, Louvain-La-Neuve.

KRAFFT J. et MAUPERTUIS M.A., (1996) : «Institutionnalisme, évolutionnisme et compétences des firmes: le point de vue de Richard Nelson », Revue d'Économie Industrielle, $\mathrm{n}^{\circ} 78$, quatrième trimestre.

LE BAS C., (1989) : « Où en est l'analyse économique du changement technique ? À propos de Technical change and economic theory », Revue d'Économie Industrielle, vol. 48, deuxième trimestre.

MARCHESNAY M., (1977) : "Sur une analyse mésoéconomique du capitalisme mondial », Revue d'Économie Industrielle, vol. 2, quatrième trimestre.

MARCHESNAY M. et MORVAN Y., (1979), « Micro, macro, méso... », Revue d'Économie Industrielle, vol. 8, deuxième trimestre.

MARCHESNAY M., (1983) : «Où en est la mésoanalyse? » in ADEFI: Économie industrielle - Problématique et méthodologie, Economica, Paris.

MARCHESNAY M., (1984), «La vulgate de l'économie industrielle: paradoxes autour d'un paradigme », Revue d'Économie Industrielle, vol. 28, deuxième trimestre.

MORVAN, Y., (1976) : Économie industrielle, Presses Universitaires de France, Paris.

MORVAN Y., (1977) : «À propos de l'économie industrielle », Revue d'Économie Industrielle, vol. 1 , troisième trimestre.

Numéro spécial sur « La statistique industrielle », (1978), Revue d'Économie Industrielle, vol.3, quatrième trimestre.

Numéro spécial «Efficacité et rentabilité », (1978), Revue d'Économie Industrielle, vol. 5, troisième trimestre.

Numéro spécial sur «Les politiques industrielles », (1983), Revue d'Économie Industrielle, vol. 23, premier trimestre.

Numéro spécial sur «Les restructurations industrielles », (1985), Revue d'Économie Industrielle, vol. 31, troisième trimestre.

Numéro spécial sur «L'investissement », (1987), Revue d’Économie Industrielle, vol. 40, deuxième trimestre.

Numéro spécial sur « Les nouvelles industries de l'information et de la communication », (1987), Revue d'Économie Industrielle, vol. 39, premier trimestre.

Numéro spécial sur «Le dynamisme des services à l'entreprise », (1988), Revue d'Économie Industrielle, vol. 43, premier trimestre.

Numéro spécial «Organisation et dynamique industrielle », (1990), Revue d'Économie Industrielle, vol. 51, premier trimestre. 
Numéro spécial «La politique de la concurrence », (1993), Revue d'Économie Industrielle, vol. 63, premier trimestre.

Numéro spécial « PME-PMI et économie industrielle », (1994), Revue d'Économie Industrielle, vol. 67, premier trimestre.

Numéro spécial « Transformations des politiques industrielles dans les années 1990 », (1995), Revue d'Économie Industrielle, vol. 71, premier trimestre.

Numéro spécial sur "L'économie industrielle de la science », (1997), Revue d'Économie Industrielle, vol. 79, premier trimestre.

Numéro spécial sur « Dynamique industrielle et contraintes environnementales », (1998), Revue d'Économie Industrielle, vol. 83, premier trimestre.

Numéro spécial «Économie de la connaissance », (1999), Revue d'Économie Industrielle, vol. 88 , deuxième trimestre.

Numéro spécial «L'économie des contrats: bilan et perspectives », (2000), Revue d'Économie Industrielle, vol. 92, deuxième et troisième trimestres.

Numéro spécial «What policies in support for R\&D? », 2001, Revue d'Économie Industrielle, vol. 94, premier trimestre.

Numéro spécial «Organisations et institutions: la centralité des règles », 2001, Revue d'Économie Industrielle, vol. 97, quatrième trimestre.

Numéro spécial «Les droits de propriété intellectuelle: nouveaux domaines, nouveaux enjeux », (2002), Revue d'Économie Industrielle, , vol. 99, deuxième trimestre.

Numéro spécial «La morphogenèse des réseaux », (2003), Revue d'Économie Industrielle, vol. 103, deuxième et troisième trimestres.

Numéro spécial «La dynamique des marchés », (2004), Revue d'Économie Industrielle, vol. 107, premier trimestre.

Numéro spécial "Connaissance et économie industrielle », (2005), Revue d'Économie Industrielle, vol. 110, deuxième trimestre.

Numéro spécial « Processus de contagion et interactions stratégiques », (2006), Revue d'Économie Industrielle, vol. 114-115, deuxième et troisième trimestres.

PALLOIX C. et RIZOPOULOS (sous la responsabilité de), (1997) : Firmes et économie industrielle, L'Harmattan, Paris.

PARENT J., (1979) : «Filières de produit, stades de production et branches d'activité », Revue d'Économie Industrielle, vol. 7, premier trimestre.

PONSSARD J.-P., (1988) : Stratégies d'entreprise et économie industrielle, MacGraw Hill, Paris.

RAINELLI M., (1977) : «À propos des découpages de l'industrie », Revue d'Économie Industrielle, vol. 1 , troisième trimestre.

RAINELLI, (1979) : «Sur les découpages de l'industrie », Revue d'Économie Industrielle, vol. 6 , quatrième trimestre.

REIX R. (1979) : " Marchés et organisations hiérarchisées. À propos d'un ouvrage de O.E. Williamson », Revue d'Économie Industrielle, vol. 8, deuxième trimestre.

REYNAUD-CRESSENT B., (1985) : «Structures industrielles et segmentation du marché du travail: théorie radicale et nouveau structuralisme », Revue d'Économie Industrielle, vol. 33, troisième trimestre.

SCHMALENSEE, R., (1988) : «Industrial Economics: An Overview», Economic Journal, Royal Economic Society, vol. 98(392), septembre.

SOLAL P. et TALLON J.-M., (2006) : «Processus de contagion et interactions stratégiques », Revue d'Économie Industrielle, vol. 114-115, deuxième et troisième trimestres.

STOFFAES C., (1980) : " Politique industrielle et filières », Revue d'Économie Industrielle, vol. 13, troisième trimestre.

TIROLE J., (1988) : The theory of industrial organization, MIT Press, Harvard.

TOLEDANO J., (1978) : « À propos des filières industrielles », Revue d'Économie Industrielle, vol. 6 , quatrième trimestre.

UMBHAUER G., (1988) : « Théorie des jeux et économie industrielle », Revue d'Économie Industrielle, vol. 45, troisième trimestre.

Varii autores, (1980) : «Approches sectorielles de la croissance et de la crise dans les cinq économies dominantes: quatre études de comparaison internationale », Statistiques et Études financières, $\mathrm{n}^{\circ} 44$. 